\title{
An improved Michelson interferometer: smoothing out the rough spots for a more effective teaching tool
}

Clarke Eastman

Clarke K. Eastman, "An improved Michelson interferometer: smoothing out the rough spots for a more effective teaching tool," Proc. SPIE 10452, 14th Conference on Education and Training in Optics and Photonics: ETOP 2017, 104521H (16 August 2017); doi: 10.1117/12.2269663

Event: 14th Conference on Education and Training in Optics and Photonics, ETOP 2017, 2017, Hangzhou, China 


\title{
AN IMPROVED MICHELSON INTERFEROMETER: SMOOTHING OUT THE ROUGH SPOTS FOR A MORE EFFECTIVE TEACHING TOOL
}

\author{
Clarke K. Eastman \\ Institute of Optics \\ University of Rochester \\ 275 Hutchinson Rd., Wilmot Building \\ Rochester NY 14627
}

\begin{abstract}
The Michelson interferometer is a classic tool for demonstrating the wave nature of light, and it is a cornerstone of the optics curriculum. But many students' experiences with this device are higher in frustration than they are in learning. That situation motivated an effort to make aligning the tool less a test of a visual acuity and patience, and more of an introduction to optics phenomena and optical engineering. Key improvements included an added beam-splitter to accommodate multiple observers, a modified telescope to quickly and reliably obtain parallel mirrors, and a series of increasing spectral-width light sources to obtain equal path lengths. This greatly improved students' chances of success, as defined by achieving "white light fringes". When presenting these new features to the students, high importance is placed on understanding why alignment was so difficult with the original design, and why the changes made alignment easier. By exposing the rationale behind the improvements, students can observe the process of problem-solving in an optical engineering scenario. Equally important is the demonstration that solutions can be devised or adapted based on the parts at hand, and that implementations only achieve a highly "polished' state after several design iterations.
\end{abstract}

\section{BACKGROUND AND MOTIVATION}

Students are learning as much theory as ever in their classes. But often they are less familiar with hands-on work compared with a generation or two ago, whether that's in a lab class or in their lives outside school. Simulation, virtual reality, augmented reality, etc. are receiving a lot of attention in the academic world. And these tools really can improve the learning experience both in the classroom and in the lab. But the work presented here was aimed directly at the underlying "reality" component of student lab experiences. Reality can give theory some credibility. Or as they say, seeing is believing. But a useful lab experience has to be more than just a lesson in coping with frustration. A good experience can complement theory and aid students' intuitive understanding of theoretical concepts. But in the lab they often encounter situations and problems that their theory classes have not prepared them for. Sometimes these situations are far ahead of the theory they're learning, and sometimes they are in a completely different subject area. Much about the lab experience is inherently multidisciplinary mechanical devices are imperfect and computer programs have bugs. The outcome of these encounters can have important consequences: Are they motivating or are they discouraging? The intent of the work described here was to make a successful lab result more likely, and to give the students some understanding of what helped them achieve that success.

\section{OUR STARTING POINT: THE MICHELSON INTERFEROMETER AND A TYPICAL ALIGNMENT EXPERIENCE}

This instrument is a cornerstone of the optics curriculum for good reason. Using it leads to only one reasonable conclusion, that light really does behave like a wave. And aligning it helps reinforce the notion that the wavelength of light is very small compared with our everyday experience. The original device became famous in 1887 after the Michelson Morley experiment, and its history is part of virtually every physics curriculum. Our interferometer is a "traditional" model that uses incoherent illumination. We don't use lasers, but some of our improvements do use components (narrow band interference filters and LEDs) that weren't available in 1887.

14th Conference on Education and Training in Optics and Photonics: ETOP 2017, edited by Xu Liu,

Xi-Cheng Zhang, Proc. of SPIE Vol. 10452, 104521H · @ 2017 ICO, IEEE, OSA, SPIE

CCC code: $0277-786 X / 17 / \$ 18 \cdot$ doi: $10.1117 / 12.2269663$

Proc. of SPIE Vol. $10452104521 \mathrm{H}-1$ 
The actual beam path in the device is shown in Figure 1. Light entering the device is divided by a beamsplitter and travels two paths. Each path ends in a mirror which reflects the light back to the beamsplitter, which then acts as a beam combiner. The combined beams are observed by one or more observers. The viewing beamsplitter cube is an added feature that will be described later. Conceptually "unfolding" the beam path eliminates the beamsplitter and compensator plate. It creates a system that is physically impossible to build but makes the alignment process easier to visualize. In this (conceptual) configuration the first goal of alignment would be to obtain nearly parallel mirrors so that interference fringes would be visible. The second goal would be to obtain equal path lengths. In this conceptual configuration, at equal path lengths the mirrors would appear to be exactly superimposed.
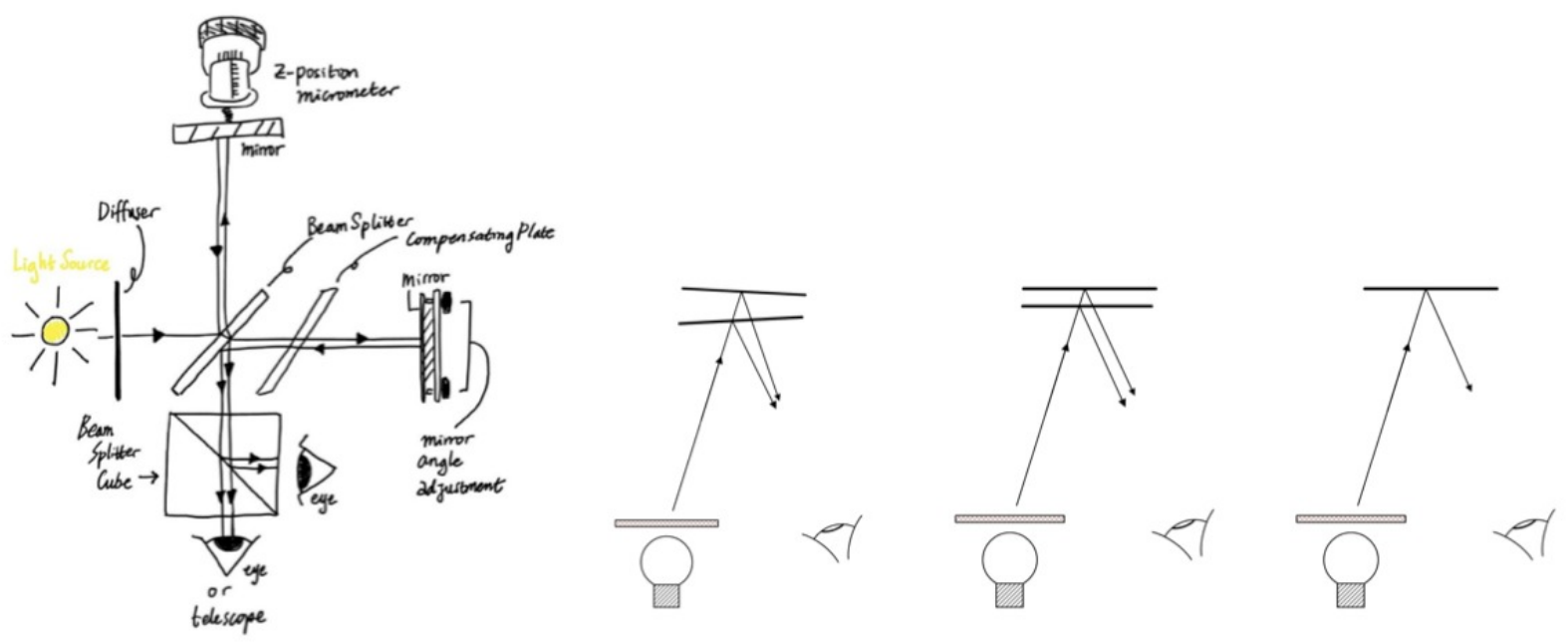

Figure 1. Different ways to describe the beam path. Left: The beam path as shown in a student lab report. The viewing beamsplitter was added as part of the work described in this paper. Right: The "unfolded" beam path, showing unaligned, partially aligned, and fully aligned systems.

A recent paper ${ }^{1}$ from the 2015 ETOP conference describes the alignment process: “...once a Michelson interferometer is misaligned, significant time can be needed for the teacher to correctly realign it". Said another way, aligning this instrument is best left to professionals. That viewpoint is both common and reasonable: the device really is hard to align. Yet at the University of Rochester, performing the alignment procedure is traditionally part of the sophomore level optics lab. A common student experience with this procedure results in frustration, and those memories tend to be persistent. Faced with this reality, one option is to drop the alignment process from the lab since it's too hard. Another is to leave alignment as a rite of passage to weed out less skilled or less motivated students. We chose to retain it, and to use engineering principles to make it easier and more instructive at the same time.

\section{THE FIRST CHALLENGE: FINDING FRINGES WITH A MONOCHROMATIC LIGHT SOURCE}

Finding interference fringes is equivalent to getting the two mirrors almost exactly "parallel" in the unfolded configuration. Fundamentally, this task is challenging because the wavelength of light is so small compared with our every day experience. In fact, "monochromatic fringes" are easy to obtain. But with a misaligned system there are far too many fringes to resolve. The real challenge is to obtain a small enough number of them that the viewer can see them. And that means getting the mirrors almost exactly parallel.

There is more than one way to visualize why interference fringes occur with monochromatic light. One can visualize (slight) mirror tilt causing path length variation across a mirror width. Or, one can visualize the two recombined beams propagating in (slightly) different directions and interfering with each other. Either way, tilting one mirror relative to the other leads to changes in a "fringe" pattern. But with the naked eye the average 
person can only see on the order of 20 fringes across a $2 \mathrm{~cm}$ wide mirror that's $250 \mathrm{~mm}$ away. With much more than that they are too close together to be resolved by most people. To get fewer than 20 fringes, the mirrors must be very close to parallel. Quantitatively that means there must be less than about 5 microns "tilt" across the mirror's width. Note that the monochromatic light we used here came from a mercury vapor lamp whose brightest spectral line is at $546 \mathrm{~nm}$.

A common method to achieve parallel mirrors is to compare the images of an object reflected by the each of the two mirrors. In our system we used a "+" target placed immediately after the light diffuser, as shown in Figure 2. When the two images are exactly super-imposed, the mirrors are parallel, or nearly so. If the images are slightly misaligned, fringes can be seen. As stated above, when mirrors are not sufficiently parallel, fringes still exist but are too close together for the viewer to resolve. In our experience roughly half the students can align the "+ target" images in our device well enough to find fringes with the naked eye. The other half cannot, at least when they first use the instrument. This last point is mentioned again near the end of the paper. For many experienced users without especially sharp vision, achieving fringes is a demonstration of skill and a point of pride. But many beginning students who succeed in this task probably accomplish it more through sharp vision than skill. In other words it's an eye test, and it shouldn't be.
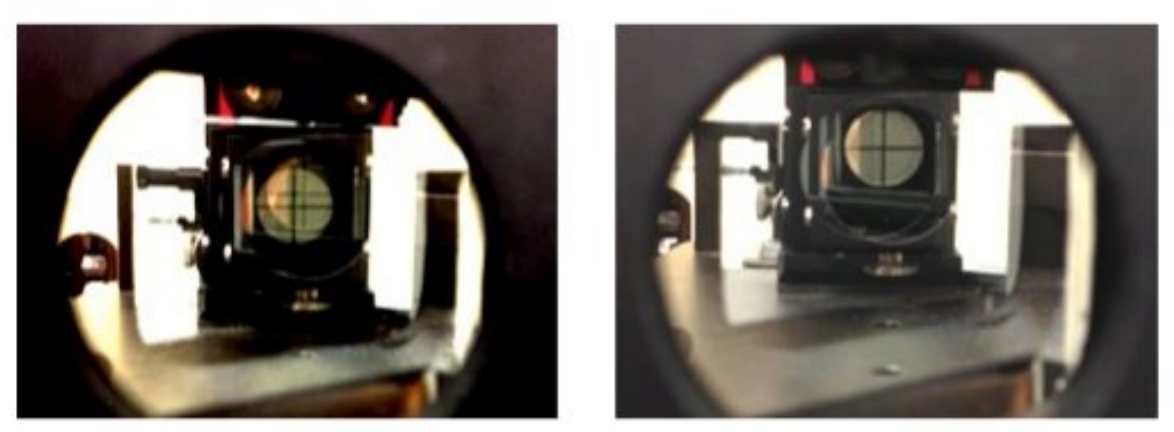

Figure 2. The view of the two "+" target images, not aligned (left) and aligned (right). When aligned, the images are superimposed and the mirrors are approximately parallel. Note that these "+" target images seem very far away. Bring them closer to the viewer is key to easily and accurately aligning them.

\section{SOLVING THE FIRST CHALLENGE: MAGNIFY THE TARGET IMAGE}

A common alignment strategy is to add a laser to the system: the student aligns the laser beams resulting from reflection by the two mirrors. We didn't do that. Instead, we looked for a simple optical device to assist the naked eye. Our need was met by a small Galilean telescope of moderate magnification, and a sufficient quantity of them was discovered in our spare parts vault. As expected these were designed to focus at a far distance. But our alignment target is only about $500 \mathrm{~mm}$ from the viewer and beyond the range of these telescopes' focus adjustment. That problem was solved by adding a small positive singlet lens (500 $\mathrm{mm}$ focal length) at the front end of the telescopes.

Ironically, a telescope had been included with the original interferometer kit. But it was intended for other purposes and its existence had passed out of collective memory. It is asserted here that in education it's sometimes $\mathrm{OK}$ to reinvent the wheel. But reading the manual is recommended - if you know it exists.

Students who used the modified telescope were able to find fringes within seconds. And when this part of the alignment process becomes reliable and repeatable, the lab experience changes. Students can see just how accurately the two target images must be aligned in order to see fringes. And they can perform the remainder of the experiment without fear of losing fringes. If they do, they can always find them again. Figure 3 below illustrates the need for a magnified view of the alignment target. The target can appear aligned by the naked eye and still not produce interference fringes. The telescope view shows that fringes are visible only when the misalignment between images is significantly less than the wire diameter $(0.5 \mathrm{~mm})$ in the "+" target. 

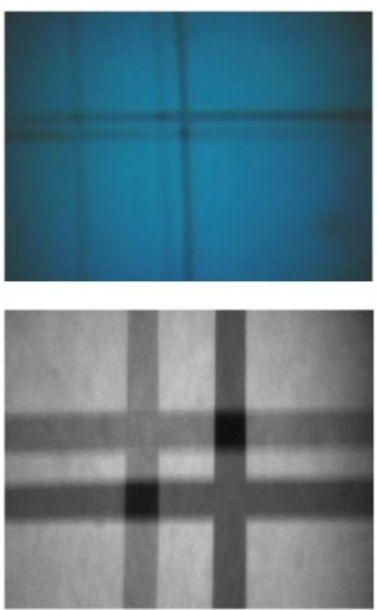
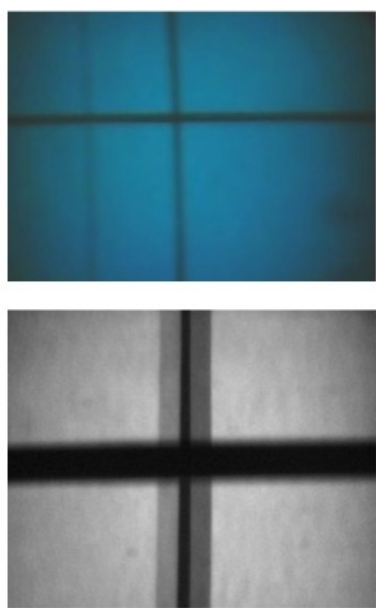
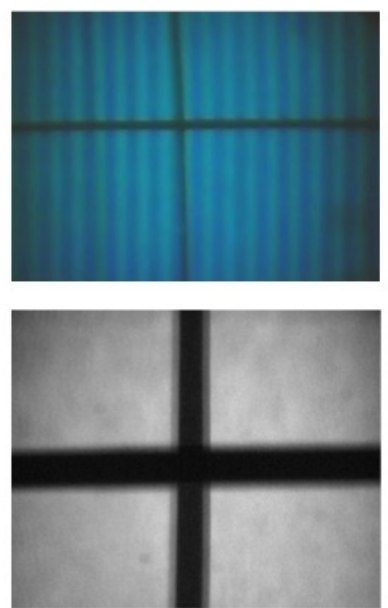
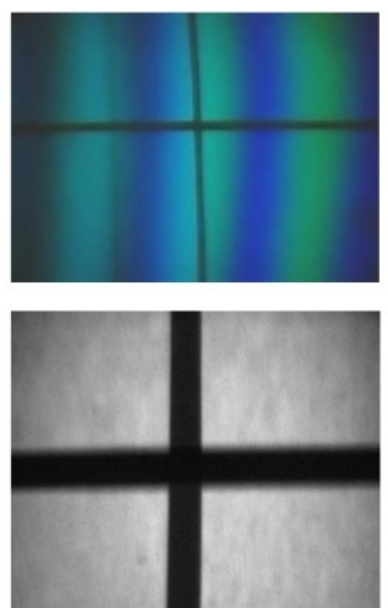

Figure 3. An Illustration of how closely aligned the target images need to be for fringes to appear. The webcam images in the top row simulate what can be seen by the naked eye. All except the left image appear to have aligned "+" patterns. (The vertical line on the far left is a reflection from another surface and does not contribute to the alignment process). But for their corresponding telescope images in the bottom row, only the image on the right is fully aligned.

As a side note, a similar telescope configuration could also be used to view magnified fringes instead of magnified alignment target images. It would let the viewer see fringes too close together to be seen by the naked eye. Although this may offer no net advantage to the method developed and described here, it leads into a discussion of fringe location, a deep and interesting subject and a perfect example of the potential of this device for teaching. In an important result from that topic, fringes from non-parallel mirrors ("fringes of equal thickness" or simply "tilt fringes") appear to be located at or near the mirrors. Referring back to the diagrams of the interferometer, the mirrors are much closer to the viewer than the alignment target is. The telescope does an excellent job magnifying the alignment target images, but it has a narrow depth of field and cannot have the alignment target and tilt fringes in focus at the same time. That problem is solved by adding a viewing beam-splitter cube. It lets one viewer align the target images with the telescope while another viewer watches for fringes to appear. In common practice this is needed only as a confirmation since the telescope virtually guarantees that fringes will be found. Figures 4 and 5 below illustrate several versions of the hardware we added.
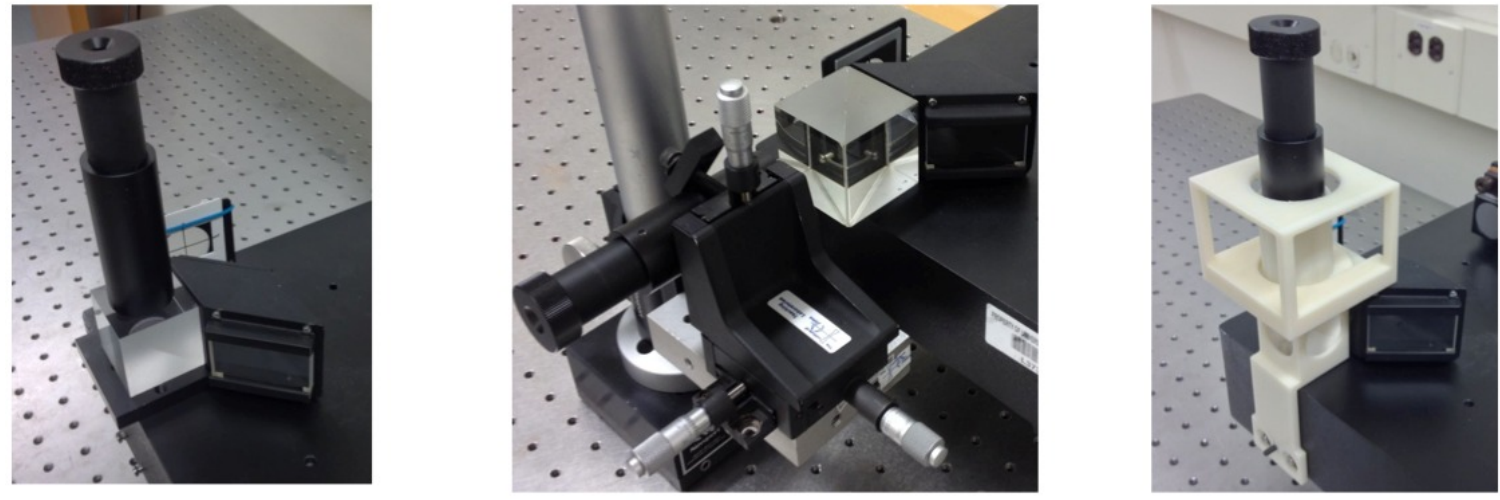

Figure 4. Three iterations of the telescope and beamsplitter configuration. Left: The initial concept was simple but not "approved for student use". Middle: The first year version. This was functional but tended to be cumbersome to set up and remove. Right: The second year version. 3-D printed parts hold the beamsplitter cube in the bottom and allow the telescope to be quickly added or removed as needed. 

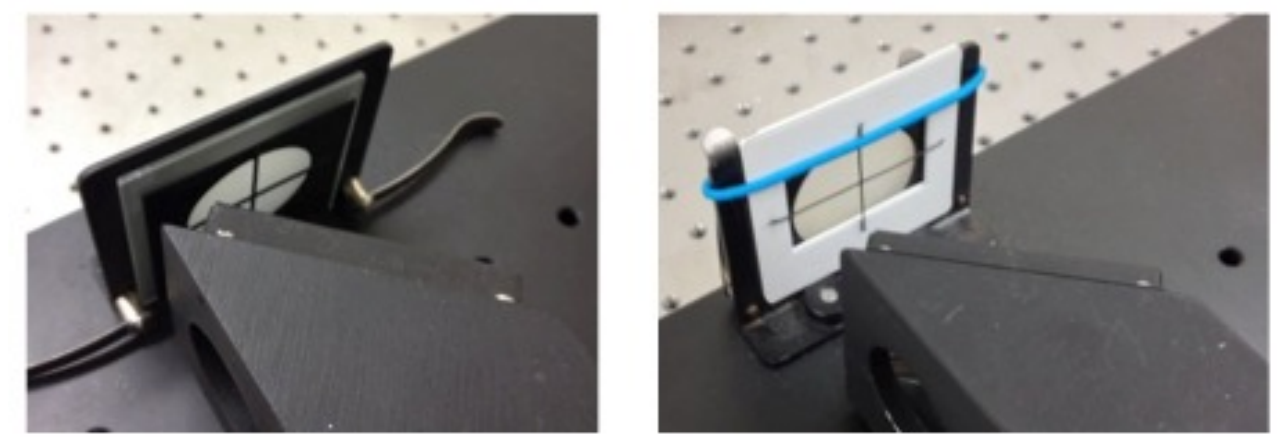

Figure 5. The original "+" alignment target (part of the original kit) is on the left and an improvised version on the right. The targets are located immediately after the diffuser. The improvised version is crude, but allows for experimentation. The wire used in the improvised target is $0.5 \mathrm{~mm}$ thick.

\section{THE SECOND CHALLENGE: ACHIEVE EQUAL PATH LENGTHS BY FINDING WHITE LIGHT FRINGES}

Like the first challenge, this has been described as a "find a needle in a haystack" scenario. And like the first challenge, attempting this without a systematic procedure is a potentially bad end memorable lab experience. Its difficulty lies in the fact that white light fringes only occur within a few microns of equal path lengths.

The traditional method is straightforward, and starts at a condition of unequal path lengths. The only requirement is knowing which path is longer and which path is shorter. Using white light illumination, the student then slowly moves one mirror toward the equal path length condition. Success is guaranteed if motion is slow enough and patience is infinite. As a quick calculation, moving a mirror a total distance of $1 \mathrm{~mm}$ at a speed of 1 micron per second will take 1000 seconds or about 17 minutes. It seems students these days just don't have the patience to do that any more! And neither does this author. But problems come when trying to make short cuts. If the mirror is moved too quickly, fringes will fly past without being seen. And if one starts too close to equal path lengths, the mirror might end up moving away from equal path lengths instead of toward them. And again no fringes will be found.

\section{SOLVING THE SECOND CHALLENGE: DIVIDE THE TASK INTO A SERIES OF SMALLER TASKS}

As stated above, white light fringes only occur within a few microns of equal path length. The usual explanation uses the concepts of coherence (temporal coherence or coherence length) and spectral width. A typical graphical illustration is shown in Figure 6 which compares fringes from broader and a narrower spectral-width sources. At equal path lengths both sources yield fringes with good contrast. But with the broad spectral-width source (e.g. white light), fringes drop off much more rapidly as path lengths become increasingly unequal. 

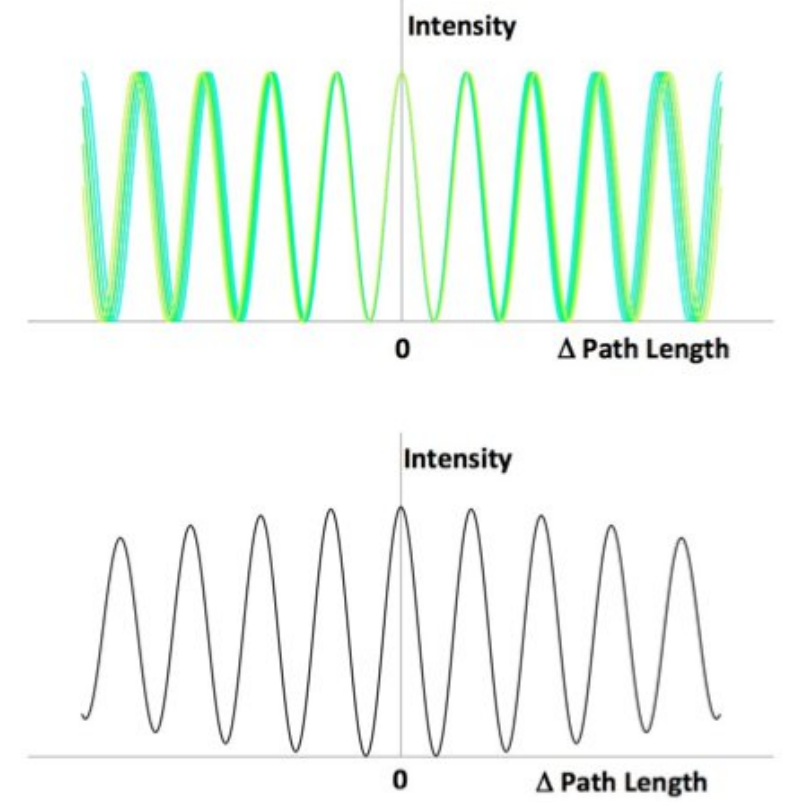
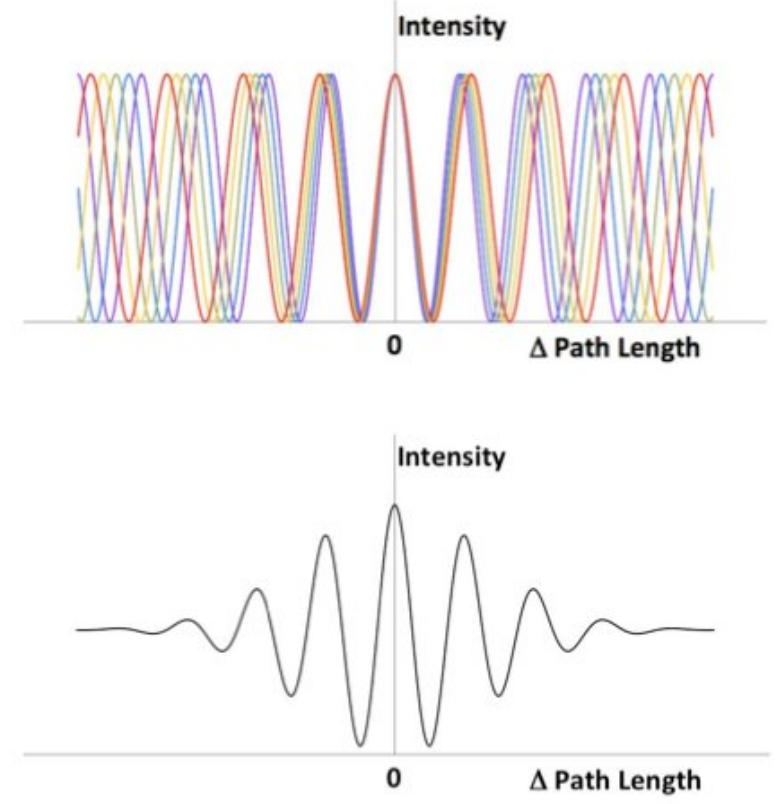

Figure 6. Simulated fringe patterns for a narrower spectral-width source (left) and broader spectral-width source (right). Fringe pattern contributions from the component wavelengths are shown on the top row, and the resulting combined fringe patterns are shown on the bottom row. Images that approximately correspond with these simulations, captured with a webcam, are shown in Figure 9.

This effect is well known, and measuring light source coherence is an important application of the Michelson Interferometer. Applying this coherence effect to the alignment process is also well known. Our contribution here may be to implement these concepts a bit differently or more systematically than what is done elsewhere. A long coherence length source (equivalently, a narrow spectral-width source) yields fringes that are visible over a wide range of unequal path lengths. Coherence length is defined quantitatively by a specified drop in fringe contrast relative to maximum contrast. Coherence length and spectral width are inversely related and defined by the equation below:

\section{$\mathbf{L} \times \Delta \lambda=\mathbf{a} \lambda^{2}$}

Where $\mathbf{L}$ is the coherence length, $\Delta \lambda$ is the spectral width, and $\lambda$ is the average wavelength. The constant "a" is not too different from 1, and depends on details of the source's spectral distribution and on the specified drop in fringe contrast.

This leads to a concept, similar to coherence length, which we call "capture range" based on what it intends to accomplish. Capture range only requires that fringes be visible at all. And so it has a similar but more qualitative relation to spectral width. Figure 7 lists the different light sources we found useful for the new alignment procedure. It graphically illustrates their associated capture ranges. Note that the $1 \mathrm{~nm}$ and $5 \mathrm{~nm}$ filters are interference filters, and need to be placed after the diffuser to work properly. 


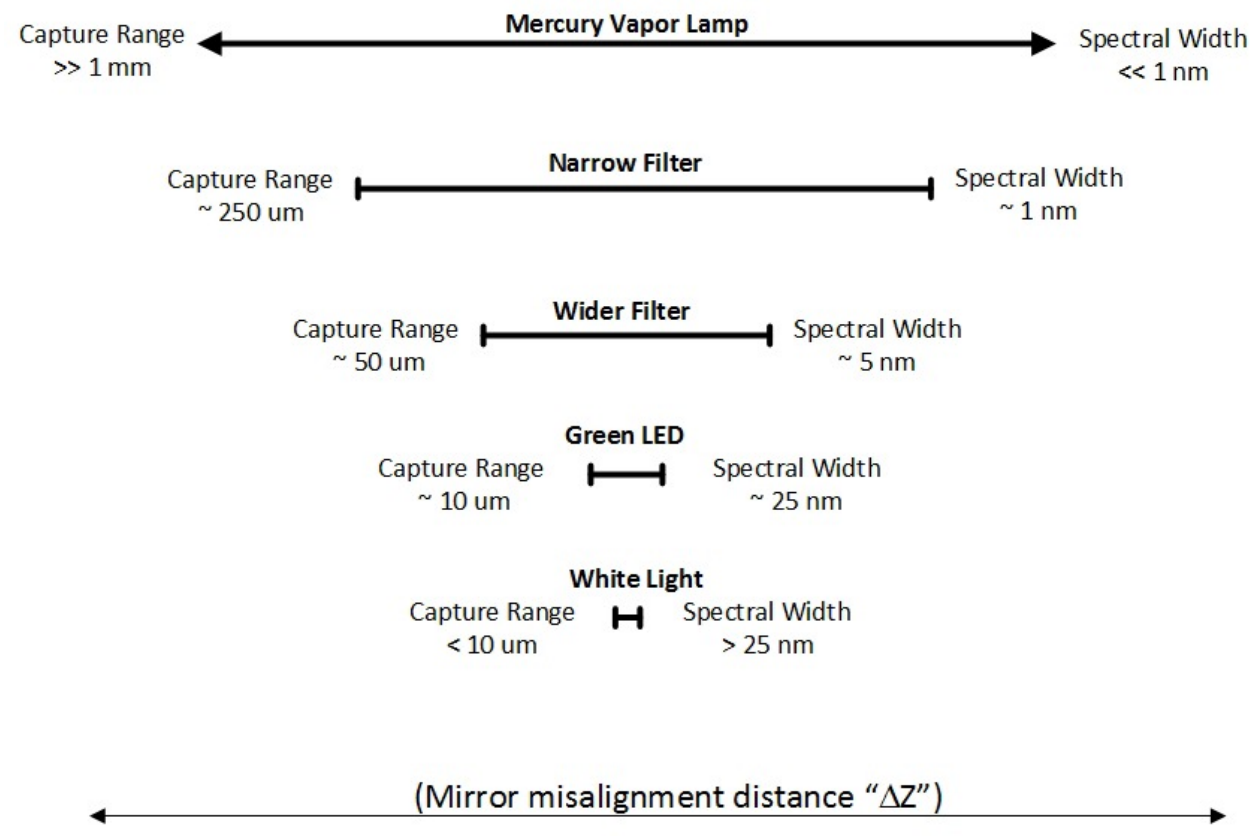

Figure 7: A graphical depiction comparing "capture ranges" for different combinations of source, filter, and measurement technique. Capture ranges are only approximate, and are not shown to scale.

The alignment process to obtain parallel mirrors (the subject of the first challenge) uses a mercury vapor lamp, whose spectral lines are extremely narrow. As a result this light source has a very long capture range. And that lets us get the mirrors exactly parallel even if mirror locations are different by many millimeters. Once parallel mirrors have been achieved they produce circular fringes. These are referred to in textbooks as "fringes of equal inclination". Interestingly, circular fringes are located at infinity, not near the mirrors as was the case for the straight-line "fringes of equal thickness". We use them in our first step toward achieving equal path lengths.
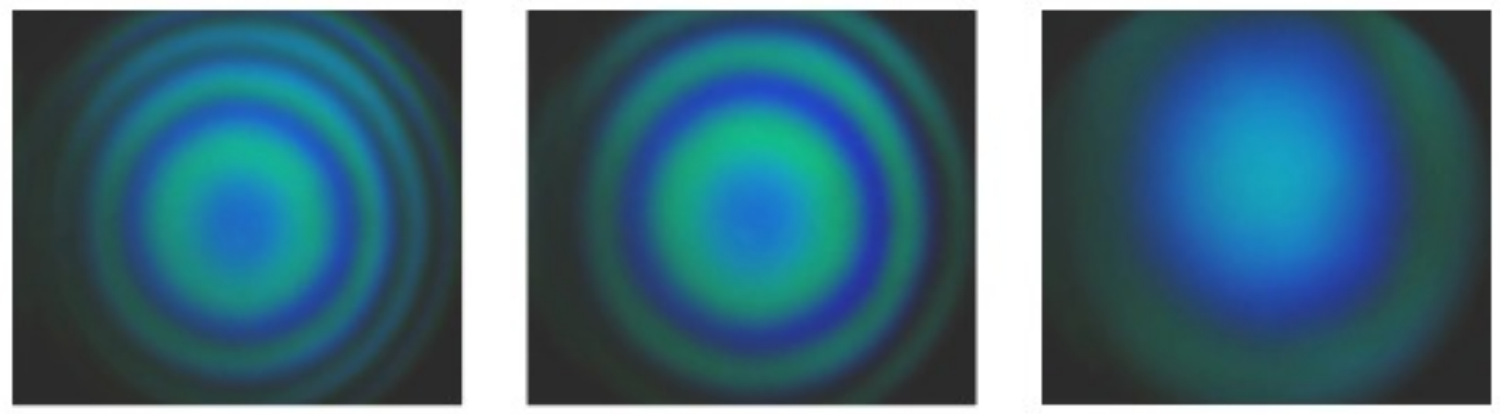

Figure 8: Circular fringes occur when the mirrors are parallel. They are useful to identify a zone around the equal path length condition, but are not a precise way to find the exact equal path condition. The path length difference in the interferometer is largest on the left and smallest on the right.

A well known and widely used property of these circular fringes is that they collapse toward their center when moving toward equal path lengths, and they expand from their center when moving away from equal path lengths. That property isn't ideal for finding the exact equal path length condition, but it can map out a zone in mirror position that contains the equal path length condition. Due to imperfections in beamsplitter and compensation plates, some interferometers have better behavior near the equal path length condition than others. This is important because a method developed on one of our interferometers may not work for all of our units! But in our experience a $1 \mathrm{~mm}$ long zone, which includes the equal path length condition, can be identified easily. 
Once this equal path length zone is identified, circular fringes are abandoned. A small amount of tilt is intentionally put back into one of the mirrors. This creates "tilt fringes" which will be easier to find in the steps that follow. Those next steps use the series of light sources shown previously in Figure 7, with gradually increasing spectral width and decreasing capture range.

The $1 \mathrm{~nm}$ and $5 \mathrm{~nm}$ spectral-width light sources were created by combining narrow band interference filters with a green LED ( $25 \mathrm{~nm}$ spectral width). The $1 \mathrm{~nm}$ wide source has a capture range of about $0.25 \mathrm{~mm}$. That makes it is nearly impossible not to find fringes if one starts at one end of the equal path length zone described above and moves in small increments toward the other end. Once these " $1 \mathrm{~nm}$ " fringes are found, the next steps proceed relatively easily. The approximate center of the $1 \mathrm{~nm}$ source's capture range is determined. Near that center point, fringes from the $5 \mathrm{~nm}$ source can be found. And near the center of that capture range one finds the $25 \mathrm{~nm}$ source fringes. And that leads to white light fringes.
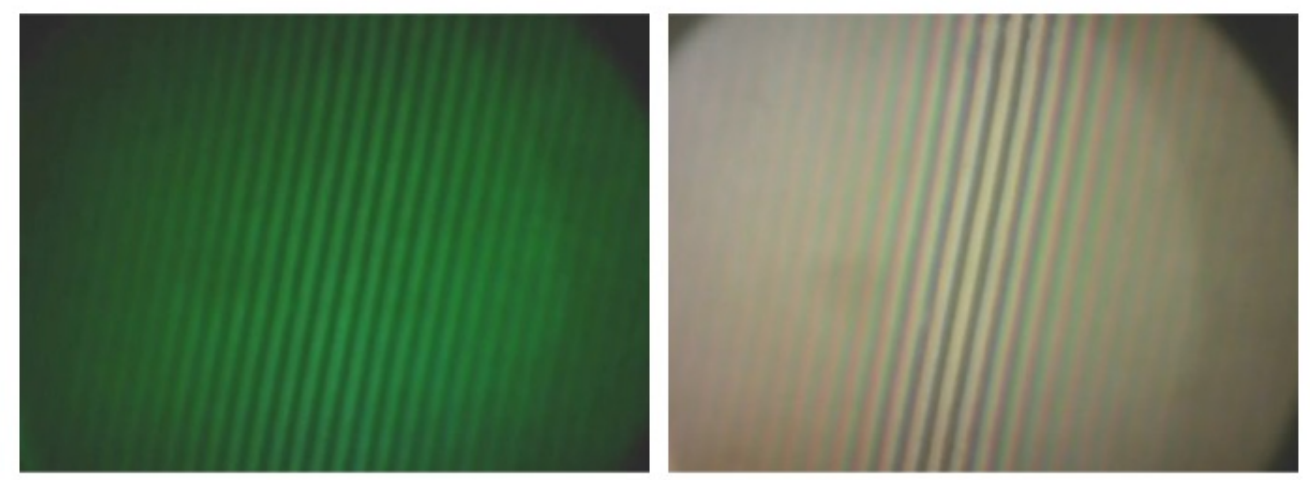

Figure 9: Comparing capture ranges of white light and green LED. The images are taken by adding a (relatively) large amount of tilt to one of the mirrors. The capture range of the green LED is actually greater than shown in the image on the left.

\section{WHAT THE STUDENTS MIGHT TAKE AWAY FROM A SUCCESSFUL LAB EXPERIENCE}

As a result of these improvements, getting white light fringes isn't so difficult anymore This leaves students more time to make their assigned measurements. And it may also lead to new measurements being assigned to them.

Students can see just how vivid the colors in white light fringes are. That may seem irrelevant to a universitylevel lab class, but the power of such things to attract and inspire students shouldn't be dismissed. They can also experience just how narrow the capture range is for white light. That was always the intent of the alignment procedure; it just didn't always happen. Having students do the alignment themselves can lead to a greater appreciation of that reality.

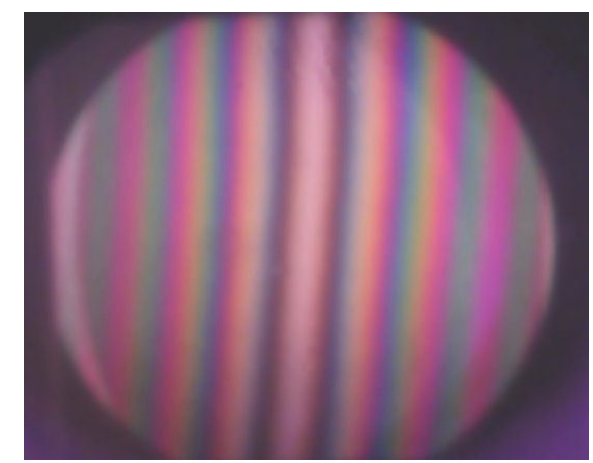

Figure 10: White light fringes as recorded with a webcam. The colors in this image don't do justice to the actual colors seen with the actual device. 
Students can see that optical engineering can make things better, easier, and maybe even fun. There were no fundamentally new techniques invented here! Instead, existing methods were applied to these problems in (perhaps) new ways. The design iterations of the telescope and viewing beamsplitter demonstrate that engineering and design typically involve gradual improvement over many product generations. Designing and 3-D printing the support hardware illustrates the potential for creative solutions.

And even using less than perfect hardware can teach a valuable lesson. Our "+" target, improvised from a $35 \mathrm{~mm}$ slide holder and electrical wire, gives students the ability to experiment with different target patterns and wire diameters.

\section{DID WE MAKE IT TOO EASY?}

For a learning experience, one viewpoint is that students should be challenged as much as possible, that tools should be just good enough to allow students to accomplish the task. Another viewpoint is that learning experiences too high in frustration are counterproductive. We believe there is truth in both of these viewpoints. This alignment procedure really has become much easier than it used to be! But the new procedure also leads to questions that can bring challenge back into the students' experience. For example, the new lab procedure required students to estimate coherence lengths of the different light sources they used. Those estimates were

then used to calculate spectral widths. And finally those spectral width calculations were compared with results from a grating spectrometer.

And although not proven yet by thorough investigation, there may have been an unexpected benefit: After using these improved methods, and seeing that the task can be done, it appeared that some students were able to accomplish the task using fewer of these improvements.

\section{ACKNOWLEDGEMENTS}

The author would like to thank Per Adamson for hardware support and many productive discussions, and WooYoun Kim for her inspired illustration of the beam path.

\section{REFERENCES}

[1] David Furió, Martin Hachet, Jean-Paul Guillet, Bruno Bousquet, Stéphanie Fleck, Patrick Reuter, Lionel Canioni, "AMI: Augmented Michelson Interferometer", paper 97930M, ETOP conference 2015 